\title{
Single-visit more effective than multiple-visit root canal treatment?
}

\author{
When individuals undergo root canal treatment for apical periodontitis, does \\ single-visit treatment result in a lower healing (success) rate than multiple-visit \\ treatment?
}

\begin{abstract}
Sathorn C, Parashos P, Messer HH. Effectiveness of single-versus multiple-visit endodontic treatment of teeth with apical periodontitis: a systematic review and meta-analysis. Int Endod J 2005; 38:347-355
\end{abstract}

Data sources Literature was searched using the Cochrane Controlled Trials Register (CENTRAL), Medline, Embase and HealthStar databases. Reference lists from identified articles were scanned and a further search made using names of authors of the identified articles. Papers that had cited these publications were also identified through the Science Citation Index to identify potentially relevant subsequent primary research.

Study selection Two reviewers scanned all titles and abstracts. An article was included if subjects had a no relevant medical history; subjects presented with mature teeth with infected necrotic root canals and radiographic evidence of periapical bone loss (as an indication of preoperative canal infection), all selected root canals had not received any endodontic treatment previously, participants underwent nonsurgical root canal treatment during the study, and the number of teeth showing radiographic evidence of healing was the outcome measure. Data extraction and synthesis Data were abstracted by two reviewers. The principal measure of treatment effect was risk difference with the fixed-effect method for combining study estimates being used to produce an overall estimate. Between-study heterogeneity was assessed using standard $\chi^{2}$ test or Q-statistic.

Results Only three randomised controlled trials (RCT) were identified and included in the review, covering a total of 146 cases. Sample size of all three studies was small. None demonstrated a statistically significant difference in healing rates. Risk differences (RD) of included studies were combined using the inverse variance-weighted method (pooled $\mathrm{RD},-6.3 \%$; $95 \%$ confidence interval, -20.3 to +7.8 ).

Conclusions Based on the current best available evidence, singlevisit root canal treatment appears to be slightly more effective than multiple visits, that is, it had a $6.3 \%$ higher healing rate. The difference in healing rate between these two treatment regimens was not statistically significant $(P=0.3809)$, however.

Address for correspondence: Harold H Messer, Department of Restorative Dentistry School of Dental Science, University of Melbourne, 711 Elizabeth Street, Melbourne, Victoria 3000, Australia. E-mail: hhm@unimelb.edu.au

\section{Commentary}

A major goal of nonsurgical root canal treatment (NSRCT) is the prevention or treatment of apical periodontitis, leading to the preservation of natural teeth. Three major factors affect the healing of apical periodontitis. First, therapeutic factors such as different regimens of NSRCT or the quality of subsequent restorations are thought to be critical in treatment outcomes. ${ }^{1-3}$ Second, host factors such as diabetes and, possibly, smoking have been associated with a decreased response to treatment. ${ }^{4}$ Third, microbial factors, such as the presence of Enterococcus faecalis, are associated with cases having a poor clinical outcome. ${ }^{5}$

There is considerable interest in the efficacy of different regimens of NSRCT on the healing of apical periodontitis, largely because the treatment protocol is under the clinician's control. Thus, this study by Sathorn and co-workers is of particular importance because it compares a one-visit treatment protocol with a multiple-visit treatment regimen that included interappointment treatment with calcium hydroxide. This intervention is based upon sound biological principles, the well-recognised antimicrobial properties of calcium hydroxide against most, but not all, endodontic microflora.

The study is a well-designed systematic review and the authors carefully identify potential design issues and limitations to the work. Unfortunately, only three RCTs were identified, leading to a relatively small total sample size. In addition, these studies provide some but not all information about other relevant factors (eg, quality of subsequent restorations, patient history of diabetes).

Given these limitations, the study does provide an important conclusion: there was no significant difference in the healing of apical periodontitis in the one-visit cases versus the multiple-visit cases with calcium hydroxide treatment. Indeed, if anything, onevisit treatment tends to have a better outcome.

These results have strong therapeutic and research implications. First, the finding of this systematic review that multiple visits with calcium hydroxide treatment does not improve upon clinical outcome provides at least a minimal level of evidence for considering one versus two appointments when planning NSRCT procedures. Although it would be optimal to base clinical recommendations upon a larger body of RCT, the authors correctly note that it is very unlikely that subsequent research would result in a greater than $10 \%$ improvement with multiple-appointment treatment. This is based upon the observation that the present study's $95 \%$ confidence interval ranges from $-7.8 \%$ (ie, favouring multiple appointments) to $+20.3 \%$ (ie, favouring the single appointment).

Secondly, this finding has considerable scientific implications. In particular, what other NSRCT regimens might further increase success in the healing of apical periodontitis? Current clinical evidence is consistent with a statistically significant but clinically small ( $\sim 10 \%$ ) difference in instrumentation/ obturation methods. In addition, the quality of the coronal restoration or the post and core appear to have a significant and perhaps larger impact on clinical outcomes. These initial studies need to be repeated by other RCT with subsequent systematic reviews. 
Finally, the biological mechanisms for persistent apical periodontitis should be studied from the perspective of developing new treatment approaches to further improve upon the success in treating this disease.

\section{Practice point}

- The review shows that multiple visits with calcium hydroxide treatment does not improve upon clinical outcome and provides at least a minimal level of evidence for considering one versus two appointments when planning NSRCT procedures.

\section{Ken M Hargreaves}

Department of Endodontics, University of Texas Health Science Center at San Antonio, San Antonio, Texas, USA
1. Farzaneh M, Abitbol S, Lawrence HP, Friedman S. Treatment outcome in endodontics - the Toronto Study. Phase II: initial treatment. J Endod 2004; 30:302-309.

2. Salehrabi R, Rotstein I. Endodontic treatment outcomes in a large patient population in the USA: an epidemiological study. I Endod 2004; 30:846-850.

3. Moshonov J, Slutzky-Goldberg I, Gottlieb A, Peretz B. The effect of the distance between post and residual gutta-percha on the clinical outcome of endodontic treatment. J Endod 2005; 31:177-179.

4. Fouad AF, Burleson J. The effect of diabetes mellitus on endodontic treatment outcome: data from an electronic patient record. J Am Dent Assoc 2003; 134:43-51; quiz 117-118.

5. Fouad AF, Zerella I, Barry I, Spangberg LS. Molecular detection of Enterococcus species in root canals of therapy-resistant endodontic infections. Oral Surg Oral Med Oral Pathol Oral Radiol Endod 2005; 99:112-118.

Evidence-Based Dentistry (2006) 7, 13-14.

doi:10.1038/sj.ebd.6400372 